\title{
The light baryon spectrum calculated with $2+1$ flavors of domain wall fermions
}

\author{
T. Blum* (RBC and UKQCD Collaborations) \\ RIKEN-BNL Research Center, Brookhaven National Laboratory, Upton, NY 11973, USA \\ Physics Department, University of Connecticut, Storrs, CT 06269-3046, USA \\ E-mail: tblumephys.uconn. edu
}

\begin{abstract}
Preliminary results for the light baryon spectrum computed in an on-going QCD simulation using $2+1$ flavors of domain wall fermions and the Iwasaki gauge action are presented and discussed. Lattice sizes $24^{3} \times 64$ and $32^{3} \times 64$ with spacings 0.114 and $\sim 0.08 \mathrm{fm}$ are used in the calculation. The physical spatial size is thus $2.74 \mathrm{fm}$ and $\sim 2.6 \mathrm{fm}$, respectively. The degenerate light quarks have masses down to $\approx 0.13$, in units of the physical strange quark mass. We present preliminary fits of the nucleon mass, including next-to-leading order heavy baryon chiral perturbation theory, which works reasonably well.
\end{abstract}

The XXVI International Symposium on Lattice Field Theory

July 14-19 2008

Williamsburg, Virginia, USA

\footnotetext{
* Speaker.
} 


\section{Introduction}

Computing the baryon spectrum is an important element of solving quantum chromodynamics (QCD), a long term goal of the high energy and nuclear physics communities. Significant progress has been made recently in the meson sector, in particular, using chiral perturbation theory to extrapolate meson masses and decay constants computed in 2+1 flavor QCD to the physical point(s). Similar progress can be hoped for in the baryon sector[1].

Here we present results, using $2+1$ flavors of domain wall fermions (DWF) and the Iwasaki gauge action, for the low-lying mass spectrum of the baryons. Nucleon $(N)$, n-star $\left(N^{*}\right)$, delta $(\Delta)$, and omega $(\Omega)$ masses are computed. The latter, consisting of three valence strange quarks, provides a robust means to set the lattice scale since the chiral extrapolation to the physical light quark point is particularly simple[2].

Building on the recent ensemble with lattice spacing $a=0.114 \mathrm{fm}[3]$ and two volumes, the RBC, UKQCD, and LHP collaborations are generating a companion ensemble with smaller lattice spacing, but with (targeted) physical volume which is the same as the larger one, $(2.74 \mathrm{fm})^{3}$. Results are presented from both ensembles but we do not yet estimate systematic errors.

\section{Simulation details}

In Table 1 the parameters of the simulations are summarized. Details of the ensemble generation can be found in [3] and in talks given at this meeting[4]. The inverse lattice spacing for the $\beta=2.13$ ensemble was found to be 1.729(3) from the $\Omega$ baryon mass[3]. The residual quark mass intrinsic to DWF, for 5th dimension size $L_{s}=16$, was found to be $m_{\text {res }}=0.00315(2)$. The values for the $\beta=2.25$ ensemble were preliminary at the time of the meeting; from the static quark potential, $a^{-1} \approx 2.42(4) \mathrm{GeV}$ and $m_{\mathrm{res}}=6.76(11) \times 10^{-4}[4]$.

The quark propagators were calculated using $16^{3}$ Coulomb gauge fixed box and gauge invariant Gaussian sources on the $24^{3}$ ensemble and Coulomb gauge fixed wall sources on the $32^{3}$ ensemble. The Gaussian source radius was $r=7$ lattice units, and 100 smearing steps were used. See [5] for details of the method.

In general the zero-momentum Baryon two point function takes the following form on an anti-periodic lattice of size $N_{t}$ in the time direction[6],

$$
C(t)=Z\left(\left(1+\gamma_{4}\right) e^{-M t}-\left(1-\gamma_{4}\right) e^{-M\left(N_{t}-t\right)}\right)
$$

where the first term represents the particle state and the second, the anti-particle. Excited state contributions have been ignored. In practice we fold the two combinations together by taking $t \rightarrow N_{t}-t$ in the second term and fit the correlation function to a single exponential.

Table 1: Simulation parameters. The size of the DWF 5th dimension is $L_{s}=16$ in each case. $m_{l}$ and $m_{s}$ refer to bare light (degenerate) and strange quark masses. $\beta=6 / g^{2}$ is the Iwasaki gauge action coupling.

\begin{tabular}{ccccc}
$\beta$ & $m_{l}$ & $m_{s}$ & size & Generator \\
\hline 2.13 & $0.005,0.01,0.02,0.03$ & 0.04 & $16^{3} \times 32,24^{3} \times 64$ & RBC, UKQCD \\
2.25 & $0.004,0.006,0.008$ & 0.03 & $32^{3} \times 64$ & LHPC, RBC, UKQCD \\
\hline
\end{tabular}


To extract the $N^{*}$ mass, for example, the excited state contribution can not be ignored. In general the correlation function has additional contributions[6],

$$
C(t)=\cdots-Z^{\prime}\left(\left(1-\gamma_{4}\right) e^{-M^{\prime} t}+\left(1+\gamma_{4}\right) e^{-M^{\prime}\left(N_{t}-t\right)}\right) .
$$

where $M^{\prime}$ denotes the first excited state with opposite parity to the ground state. We again fold the correlation function and fit to a singe exponential, but in the region close to $t=N_{t}$.

Several steps have been taken to achieve high statistics. Measurements were made on as many as four time slices per configuration, usually separated by 16 lattice units, but occasionally fewer. The measurements were made as frequently as every $10 t h$ trajectory and were averaged into blocks spanning 40 monte-carlo time units. See Table 2. The large number of configurations used for the $m_{l}=0.005,24^{3}$ ensemble were needed for a recent calculation of the nucleon axial charge, $g_{A}[8]$. To make this possible, a "doubled" Gaussian source was used where each pair of sources were separated by 32 time units (half the lattice).

Table 2: Measurement summary.

\begin{tabular}{cccccc}
\hline lattice size & $m_{l}$ & source type & correlators & source time slices & \# measurements \\
\hline \hline $24^{3}$ & 0.005 & Gauss $(r=7)$ & nucleon & $0,8,16,19,32,40,48,51$ & $932^{*}$ \\
$24^{3}$ & 0.005 & Box & decuplet & 0,32 & 90 \\
$24^{3}$ & 0.01 & Gauss $(r=7)$ & nucleon & $0,8,16,19,32,40,48,51$ & 357 \\
$24^{3}$ & 0.01 & Box & decuplet & 0,32 & 90 \\
$24^{3}$ & 0.02 & Gauss $(r=7)$ & nucleon & $0,8,16,19,32,40,48,51$ & 99 \\
$24^{3}$ & 0.02 & Box & decuplet & 0,32 & 43 \\
$24^{3}$ & 0.03 & Gauss $(r=7)$ & nucleon & $0,8,16,19,32,40,48,51$ & 106 \\
$24^{3}$ & 0.03 & Box & decuplet & 0,32 & 44 \\
$32^{3}$ & 0.004 & Wall & decuplet, nucleon & $0,16,32,48$ & 116 \\
$32^{3}$ & 0.006 & Wall & decuplet, nucleon & $0,16,32,48$ & 245 \\
$32^{3}$ & 0.008 & Wall & decuplet, nucleon & $0,16,32,48$ & 179 \\
\hline
\end{tabular}

${ }^{*}$ Doubled sources, separated by 32 time slices in a pair

\section{Results}

The fitted masses for the octet and decuplet baryons are summarized in Table 4. A few representative effective mass plateaus are shown in Figure 1. The effective masses plateau from above for the Gaussian source, below for the box and wall sources. For the $24^{3}$ lattice where both box and Gaussian sources are available, the zero momentum ground state dominates sooner for the former (top two panels of Figure 1). The heavier states also plateau later. The fitted masses are shown in Figure 2, including a rather noisy estimate of the $N^{*}$ spectrum for the $32^{3}$ lattice. The time slice ranges for the fit were chosen to minimize the statistical error while still maintaining a reasonable $\chi^{2}$.

The calculations are still on-going. In the $32^{3}$ case for the lightest mass, 0.004 , a nonmonotonic behavior in the $\Omega$ mass is visible. We expect this is due to insufficient statistics and 
are continuing to measure correlators on this ensemble. Obviously, better statistics are also required for the excited baryons as well. We have not even attempted to extract the first excited state in the positive parity channel, and do not quote results for the $N^{*}$.

Table 3: Baryon masses, $24^{3}$ lattice. $\Omega$ baryon values are from [3].

\begin{tabular}{cccccc}
\hline state & $m_{l}$ & $m_{\text {val }}$ & fit range & mass & $\chi^{2} /$ dof \\
\hline$N$ & 0.005 & 0.005 & $6-12$ & $0.6713(41)$ & $1.3(1.0)$ \\
$\Delta$ & & & $4-12$ & $0.8647(107)$ & $0.40(58)$ \\
$\Omega$ & & 0.03 & $8-14$ & $0.9647(65)$ & $0.5(7)$ \\
& & 0.04 & & $1.016(51)$ & $0.6(8)$ \\
$N$ & 0.01 & 0.01 & $8-12$ & $0.7068(40)$ & $1.6(1.5)$ \\
$\Delta$ & & & $4-12$ & $0.8905(76)$ & $0.79(73)$ \\
$\Omega$ & & 0.03 & $8-14$ & $0.9809(88)$ & $1.1(1.1)$ \\
& & 0.04 & & $1.033(60)$ & $1.2(1.2)$ \\
$N$ & 0.02 & 0.02 & $7-12$ & $0.8033(65)$ & $1.0(1.7)$ \\
$\Delta$ & & & $4-12$ & $0.9626(76)$ & $1.4(1.0)$ \\
$\Omega$ & 0.02 & 0.03 & $8-14$ & $1.030(11)$ & $1.7(1.6)$ \\
& & 0.04 & & $2.0(1.8)$ & $1.074(10)$ \\
$N$ & 0.03 & 0.03 & $6-12$ & $0.8985(65)$ & $0.95(1.64)$ \\
$\Delta$ & & & $4-12$ & $1.0264(105)$ & $0.50(61)$ \\
$\Omega$ & 0.03 & 0.03 & $8-14$ & $1.040(10)$ & $1.4(1.7)$ \\
& & 0.04 & & $1.082(6)$ & $1.8(2.2)$ \\
\hline
\end{tabular}

\section{Analysis and discussion}

The fitted nucleon mass for the $24^{3}$ lattice is in reasonable enough shape that we attempt to fit its quark mass dependence and extrapolate to the physical point. The results from a simple linear fit are displayed in Figure 3 (solid line). The $\chi^{2}$ of the fit is a bit high. Still, it seems to give a reasonable representation of the data points but significantly overshoots the well-known experimental value. A more sophisticated fit, using heavy baryon chiral perturbation theory [7], is also attempted and shown in the figure (dashed line). This time, the fit nicely extrapolates close to the physical value, but the $\chi^{2}$ of the fit is large! (and we only fit the lightest three points). One may worry that the lighter baryon masses suffer finite volume effects which spoil the continuum form. Since the leading correction is proportional to $g_{A}$, the axial charge, and since $g_{A}$ may also suffer from large finite volume effects [8], one can attempt to use the lattice values of $g_{A}$ at each quark mass in the fit formula, instead of the experimental result. The fit is shown in the right panel of Figure 3 where for convenience we first subtract the known leading correction proportional to $g_{A}$ from the nucleon mass, and then fit this shifted mass. We also shift the mass using the experimental value of $g_{A}$ for comparison (this amounts to the same fit shown in the left panel). One can see the value of $\chi^{2}$ is significantly reduced by using the value of $g_{A}$ computed in finite volume, though it is still high. 
Table 4: Baryon masses, $32^{3}$ lattice.

\begin{tabular}{cccccc}
\hline state & $m_{l}$ & $m_{\text {val }}$ & fit range & mass & $\chi^{2} /$ dof \\
\hline$N$ & 0.004 & 0.004 & $6-12$ & $0.4670(67)$ & $0.95(0.94)$ \\
$\Delta$ & & 0.004 & $5-12$ & $0.5813(74)$ & $0.23(0.41)$ \\
$\Omega$ & & 0.025 & $11-16$ & $0.7268(79)$ & $1.3(1.2)$ \\
& & 0.03 & $11-16$ & $0.7548(67)$ & $1.2(1.2)$ \\
$N$ & 0.006 & 0.006 & $6-12$ & $0.4773(33)$ & $0.59(76)$ \\
$\Delta$ & & 0.006 & $6-12$ & $0.6084(52)$ & $1.7(1.3)$ \\
$\Omega$ & & 0.025 & $10-14$ & $0.7163(44)$ & $0.95(1.17)$ \\
& & 0.03 & $10-14$ & $0.7442(39)$ & $0.99(1.18)$ \\
$N$ & 0.008 & 0.008 & $7-12$ & $0.5034(42)$ & $0.90(99)$ \\
$\Delta$ & & 0.008 & $7-12$ & $0.6275(57)$ & $0.62(87)$ \\
$\Omega$ & & 0.025 & $10-14$ & $0.7253(53)$ & $1.06(1.22)$ \\
& & 0.03 & $10-14$ & $0.7534(45)$ & $1.28(1.36)$ \\
\hline
\end{tabular}

In our previous study of the light meson spectrum on the $24^{3}$ lattice[3], the $\Omega$ baryon mass was used to set the lattice spacing because being made of three strange quarks, it has a particularly simple (sea quark) chiral extrapolation[2]. The chiral extrapolation for the valence quark mass equal to the strange sea quark, and another value close to it, is shown in Figure 4. For the $32^{3}$ case, it appears the statistics are insufficient at this stage for a reliable extrapolation. Using all three points, the slope is flat. If the lightest point is dropped (because we don't trust the statistics), then the value in the chiral limit is significantly reduced.

The results presented here are preliminary. We are currently increasing the statistics on the $32^{3}$ ensemble. Further analysis of the chiral limit of the baryon masses and the extraction of excited state masses will follow.

Acknowledgements We thank the US Department of Energy, RIKEN, and USQCD for the support necessary to carry out this research. This research was also supported by US DOE grant DE-FG02-92ER40716. Computations were carried out on the DOE and RBRC QCDOC supercomputers at BNL.

\section{References}

[1] See talks at this meeting, including the plenary talk by A. Walker-Loude.

[2] D. Toussaint and C. T. H. Davies, Nucl. Phys. Proc. Suppl. 140, 234 (2005) [arXiv:hep-lat/0409129].

[3] C. Allton et al., arXiv:0804.0473 [hep-lat].

[4] See the talks of E. Scholz and S. Syritsyn.

[5] F. Berruto, T. Blum, K. Orginos and A. Soni, Phys. Rev. D 73, 054509 (2006).

[6] S. Sasaki, T. Blum and S. Ohta, Phys. Rev. D 65, 074503 (2002).

[7] E. E. Jenkins and A. V. Manohar, Phys. Lett. B 259, 353 (1991).

[8] T. Yamazaki et al. [RBC+UKQCD Collaboration], Phys. Rev. Lett. 100, 171602 (2008). 

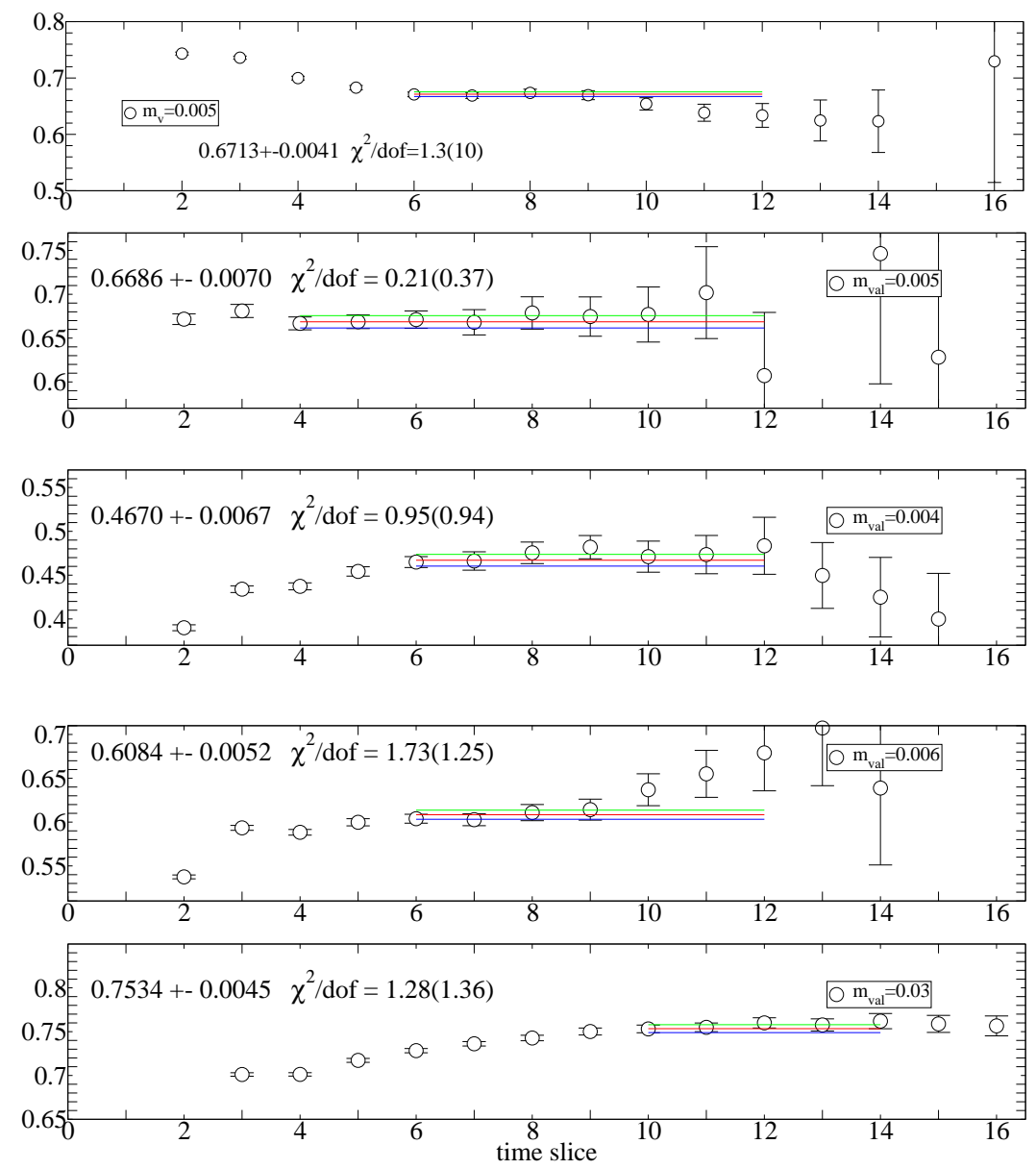

Figure 1: A sample of baryon effective masses. From the top, nucleon, $m_{l}=0.005,24^{3}$, Gaussian source; same as first panel, but box source; nucleon, $m_{l}=0.004,32^{3} ; \Delta, m_{l}=0.006,32^{3}$; and $\Omega, m_{l}=0.008,32^{3}$. 

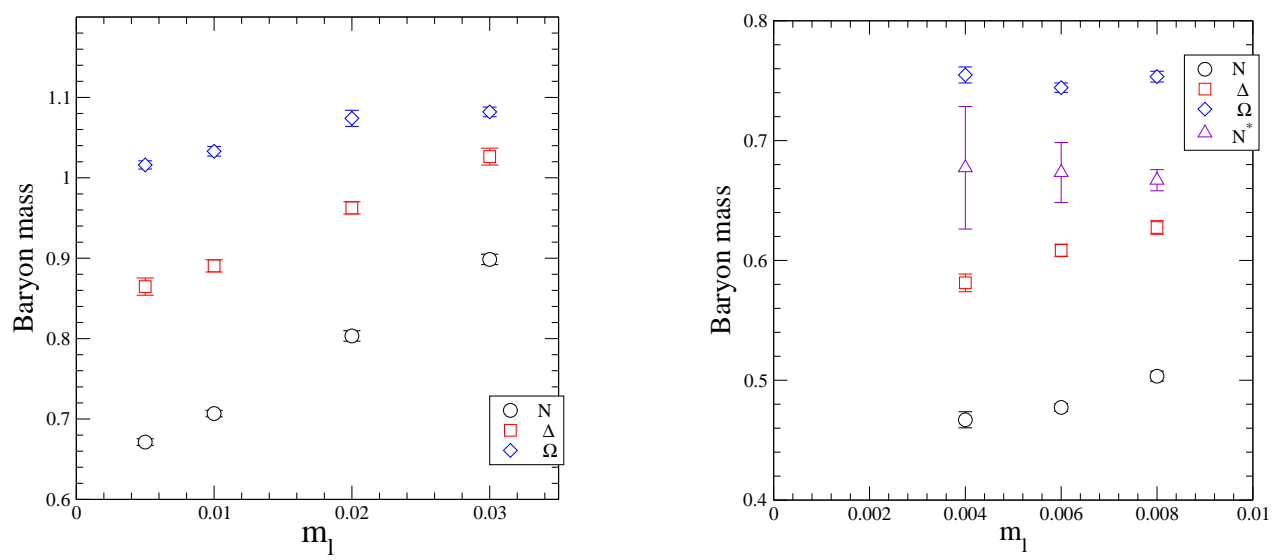

Figure 2: Baryon spectrum computed on the $24^{3}$ (left) and $32^{3}$ ensembles.
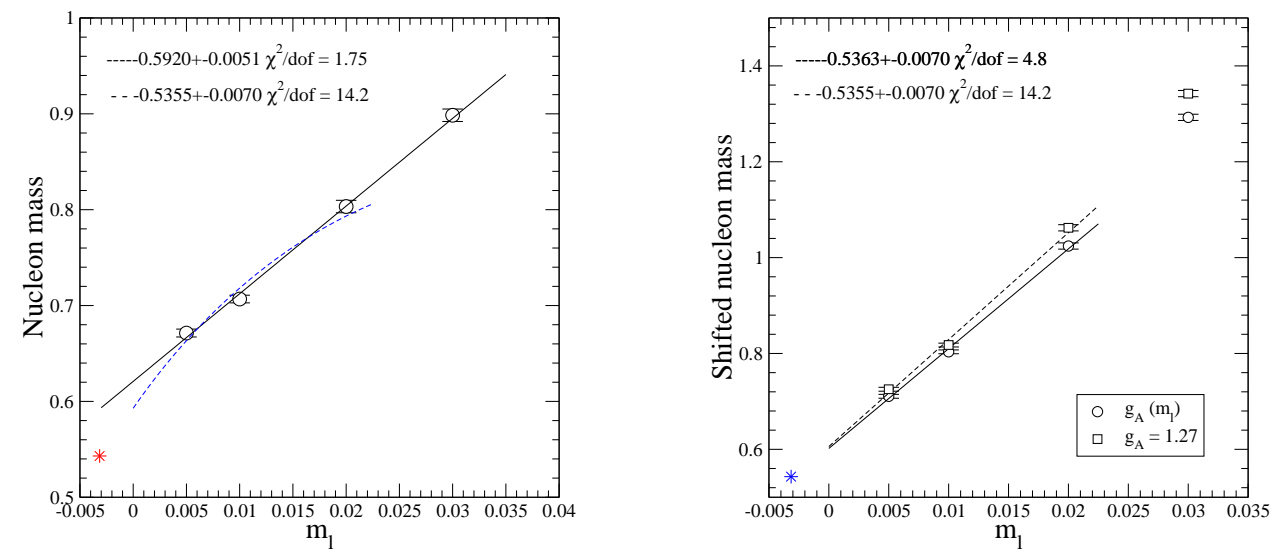

Figure 3: Chiral extrapolations of the nucleon mass. A linear fit (left panel) overshoots the experimental value (burst). The Heavy Baryon $\chi$ perturbation theory does better, but the $\chi^{2} /$ dof of the fit is high. After subtracting the finite volume effect due to the axial charge, $g_{A}$, the fit is much better (right panel).
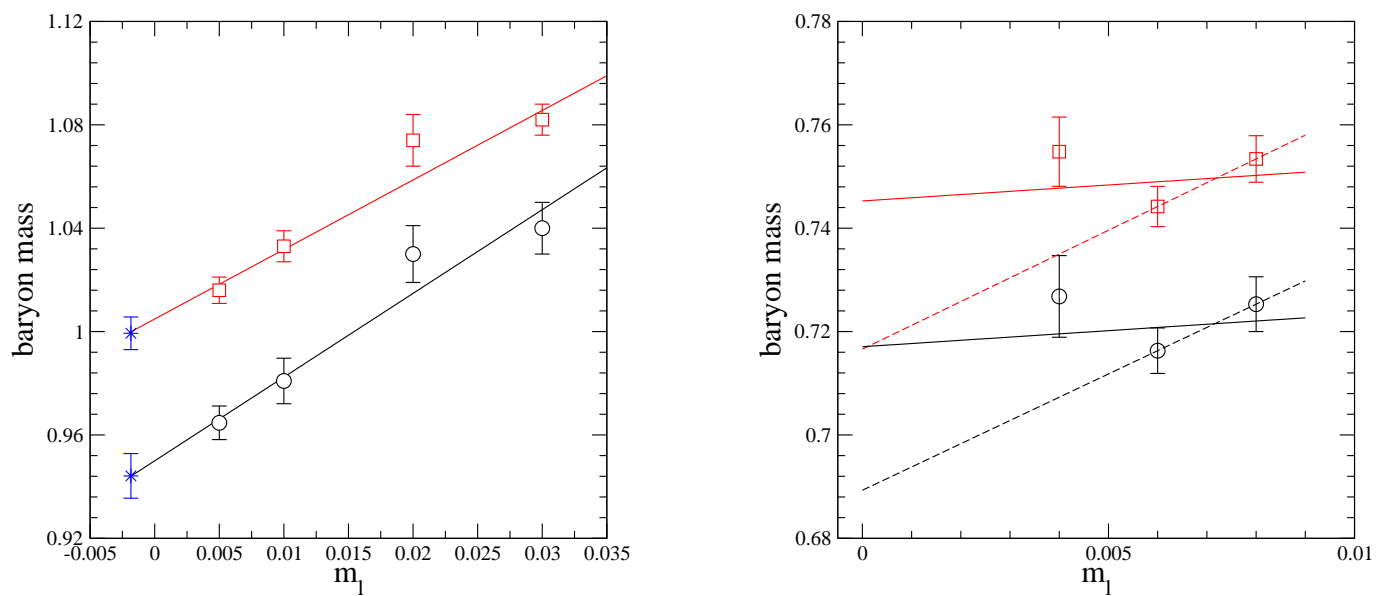

Figure 4: $\Omega$ mass chiral extrapolation. The left panel $\left(24^{3}\right)$ is from [3]. 